\title{
1 Effects of Very High Radiation on SiPMs
}

\author{
A. Heering ${ }^{a *}$, Yu. Musienko ${ }^{\mathrm{a}, \mathrm{b} *}$, R. Ruchti $^{\mathrm{a}}$, M. Wayne $^{\mathrm{a}}$, A. Karneyeu ${ }^{\mathrm{b}}$ and V. \\ Postoev \\ a. University of Notre Dame, Notre Dame, IN 46556, USA \\ b. Instutute for Nuclear Research RAS, pr. 60-letiya Oktyabrya 7a, 117312 Moscow, Russia \\ E-mail: Adriaan.Heering@cern.ch \\ E-mail: Iouri.Musienko@cern.ch
}

9 ABSTRACT: During the last 5 years we have successfully completed R\&D for the 10 instrumentation of silicon photo multipliers (SiPMs) for the CMS HCAL Phase 1 upgrade in 11 2018. Much focus was put on radiation damage during these years. For the HCAL we expect a 12 maximum total dose of $10^{12} \mathrm{n} / \mathrm{cm}^{2}$ for a total lifetime integrated luminosity of $3000 \mathrm{fb}^{-1}$. Good 13 correlation between cell size and performance with high radiation was found during this R\&D. 14 To evaluate the possibility of using the SiPMs in the wider CMS environment we have exposed 15 the current state of the art smallest cell SiPMs to radiation of $610^{12} \mathrm{p} / \mathrm{cm}^{2}$ in $62 \mathrm{MeV}$ LIF beam 16 line in 2014 at UCL Belgium and up to $1.310^{14} \mathrm{p} / \mathrm{cm}^{2}$ in the CERN PS $23 \mathrm{GeV}$ proton beam in 17 late 2014. The SiPM's main parameters were measured before and after irradiation. Here we 18 report on the effects of noise increase and breakdown voltage shift due to the extremely high 19 dose.

21 KEYWORDS: Silicon photomultiplier, MPPC, GAPD, CMS, Radiation damage.

PACS: 85.60.Bt, 85.60.Ha, 61.80.Hg

* Corresponding authors. 


\section{Introduction}

During the CMS Phase I upgrade R\&D we performed many radiation measurements on SiPMs from different companies [1-9]. We observed the expected current increase due to defects created by radiation in the silicon. We compared this current increase with the data from existing PIN diodes. As a reference we are using the data from reference[10]. The authors plotted the current increase in silicon PIN diodes per volume versus the $1 \mathrm{MeV}$ equivalent neutron dose and they found the same slope of $3.5^{*} 10^{-17} \mathrm{~A} / \mathrm{cm}$ for all different $\mathrm{p}$-type and n-type PIN diodes at $\mathrm{T}=20^{\circ} \mathrm{C}$. We expected to see the similar damage in SiPMs.

Due to high gain in SiPMs we can see the current as single photo electron increase. This implies that we can write the expected dark count rate (DCR) in the SiPM due to radiation as single photo-electron noise due to defects generated by radiation as :

$$
\mathrm{DCR}=1 / \mathrm{q} * \Phi_{\mathrm{eq}} * \mathrm{~V} * \mathrm{~S} * \mathrm{GF} * \mathrm{P}_{(\mathrm{E})}
$$

Where $\Phi_{\mathrm{eq}}$ is the $1 \mathrm{MeV}$ neutron equivalent dose per $\mathrm{cm}^{2}, \mathrm{~V}$ is the effective volume per $\mathrm{cm}^{3}, \mathrm{~S}$ is the silicon radiation constant of $3.5^{*} 10^{-17} \mathrm{~A} / \mathrm{cm}$ found in PIN diodes, GF is the geometric factor and $\mathrm{P}(\mathrm{E})$ is the geiger mode probability. To compare data at different overvoltage and different layouts of SiPMs of various manufactures we use the $\mathrm{PDE}=\mathrm{GF}^{*} \mathrm{P}_{(\mathrm{E})}$ to normalize them to each other [2]. In general we expect the SiPMs to be much more radiation resistant than PIN diodes because they have a much smaller epitaxial layer in the order of a few microns than PIN junctions that are typically 100 to $300 \mu \mathrm{m}$. Experimentally we found much higher values in the different SiPMs than expected for a $2 \mu \mathrm{m}$ epitaxial layer. We therefore think that the effective volume is much larger (10 to 20x) because the dark noise generation rate in APDs/SiPMs is dominated by high electric field effects which causes tunneling effects [1112]. Fig. 1 shows the experimental noise increase found in a 15 micron Hamamatsu (HPK) SiPM with $30 \%$ PDE (at 3V over-voltage) at different temperatures vs. an "ideal" SiPM with noise increase using the $3.5^{*} 10^{-17} \mathrm{~A} / \mathrm{cm}$ slope found in PIN diodes.

In addition to the higher noise we found that if the DCR per SiPM cell approaches the recovery time of the cell, a blocking effect will prevent the cell to respond to incoming photons during normal operation. Typically 50 micron cell pitch SiPMs become completely saturated between $10^{11}$ and $10^{12} \mathrm{n} / \mathrm{cm}^{2}$ at room temperature depending on their recovery time and GF [8]. By using much smaller cell pitch devices this effect can be suppressed because the dose per cell is much smaller. In addition, the recovery time of a small cell SiPM is much faster because it is linear with the cell capacitance. To further investigate the use of SiPMs in even higher radiation environments we exposed state of the art 10 and 12 micron cell devices to very high doses of $6^{*} 10^{12}$ at the LIF in Belgium and $10^{14}$ protons at the CERN PS beam line.

2. Radiation test to $1.2 * 10^{13} 1 \mathrm{MeV}$ equivalent $\mathrm{n} / \mathrm{cm}^{2}$

In March 2014 we exposed an array of 8 channels of $2.8 \mathrm{~mm}$ diameter with 10 micron cell HPK SiPMs to a total dose of $6 * 10^{12} 62 \mathrm{MeV}$ protons $/ \mathrm{cm}^{2}$ in the LIF facility at UCL Belgium. This corresponds to a total dose of $1.2 * 10^{13} 1 \mathrm{MeV}$ eq. $\mathrm{n} / \mathrm{cm}^{2}$. The $8 \mathrm{HPK}$ SiPMs in the array had a PDE of $12 \%$ at 3 Volt over-voltage. The current increase due to the radiation was measured after annealing and was uniform (better than 20\%) for all 8 channels. Fig. 2 shows the 
dark current of one of the channels after radiation and annealing at $\mathrm{T}=23^{\circ} \mathrm{C}$ and $\mathrm{T}=-29^{\circ} \mathrm{C}$. We found the RMS noise in a $25 \mathrm{~ns}$ gate at 3 Volt over-voltage equivalent to 16 p.e and 2.5 p.e $/ \mathrm{mm}^{2}$ respectively. The 16 p.e. $/ \mathrm{mm}^{2}$ is consistent with the projected data in figure 1 where we find 20 p.e. $/ \mathrm{mm}^{2}$ at $\mathrm{T}=20^{\circ} \mathrm{C}$. The slightly lower value can be explained by the lower GF of the 10 micron vs. the 15 micron cell. No other side effects like saturation were found except a breakdown voltage shift of about $200 \mathrm{mV}$ compared with the preradiation.

\section{Radiation test to $2.2 * 10^{14} 1 \mathrm{MeV}$ equivalent $\mathrm{n} / \mathrm{cm}^{2}$}

In December 2014 we repeated the radiation of an almost identical HPK array in the CERN PS proton beam with a requested total dose of $\sim 10^{14} \mathrm{p} / \mathrm{cm}^{2}$. In addition we radiated a 1 $\mathrm{mm}^{2}$ FBK 12 micron HD sample. Both samples had a very fast recovery time of 6 ns to avoid saturation. The HPK array had a quartz window and the FBK sample had no epoxy to avoid problems with darkening of the encapsulation. The CERN PS Beam has a $5 \times 5 \mathrm{~mm}^{2}$ beam spot and drops of gaussian. We focused the beam on the second channel of the 10 micron cell HPK Array. Each channel is a $2.8 \mathrm{~mm}$ diameter SiPM spaced $4 \mathrm{~mm}$ apart, so only channel 2 would get the full dose and a quick drop of radiation was expected for the other channels.

To calibrate the real dose we placed a $5 \times 5 \mathrm{~mm}$ calibrated linear APD (as used in the CMS ECAL) and a 3.6x3.6 mm PIN diode (HPK 1226-01) in the setup. All four devices were radiated in the same run. For the 5x5mm APD we found a final dose of $2.2 \pm 0.1 * 10^{14} 1 \mathrm{MeV}$ eq. $\mathrm{n} / \mathrm{cm}^{2}$ and for the smaller PIN diode we found a final dose of $2.5 \pm 0.5 * 10^{14} 1 \mathrm{MeV}$ eq. $\mathrm{n} / \mathrm{cm}^{2}$.

Fig. 3a shows the current vs voltage of the 8 channels of the HPK array at $\mathrm{T}=23^{\circ} \mathrm{C}$. Besides a large shift in bias voltage we found the device is operational after this radiation. The breakdown shift is almost 4 Volt. Fig $3 \mathrm{~b}$ shows the breakdown voltage vs. the current measured at $20 \mathrm{~V}$ for the pixels with different dose. If we assume that the increase in current at gain 1 (far below breakdown) is proportional with the dose, we can conclude that the breakdown shift is linear with the applied dose.

A second effect we experienced was a drop in gain due to the self heating of the device. We experienced gain drop at higher over-voltages where the currents exceeded $300 \mu \mathrm{A} / \mathrm{mm}^{2}$. In this case the breakdown voltage shifts, resulting in lower gain and eventually self regulating behavior. Without cooling operation, with small gain and PDE loss is still possible up to $1 \mathrm{~V}$ over-voltage. If we compare the current at $1 \mathrm{~V}$ over-voltage of channel $2(2 \mathrm{~mA})$ with the current after $1.2 * 10^{13}$ of $100 \mu \mathrm{A}$ at $1 \mathrm{~V}$ in figure 2 we find again the expected linear behavior dark current increase vs. the dose

Fig. 4 shows the data for the $1 \mathrm{~mm}^{2} \mathrm{FBK}$ diode after one small irradiation of $2 * 10^{11} \mathrm{n} / \mathrm{cm}^{2}$ at LIV and a large dose of $2.2 * 10^{14} \mathrm{n} / \mathrm{cm}^{2}$ at the CERN PS at $\mathrm{T}=23^{\circ} \mathrm{C}$. No effect except for the leakage current and noise increase was measured after the first small dose. We found 2 p.e. noise after this first radiation in $25 \mathrm{~ns}$ gate at $1.5 \mathrm{~V}$ over-voltage. The PDE of the sample was $10 \%$ at $520 \mathrm{~nm}$ at V-VB $=1.5 \mathrm{~V}$. Fig. 4a shows the LED response of the FBK SiPM after radiation vs bias voltage. We found a much smaller bias voltage shift of only $0.5 \mathrm{~V}$. Fig $4 \mathrm{~b}$ shows the relative response after correction of this bias voltage shift. We found that the signal is dropped by $50 \%$ at $\mathrm{V}-\mathrm{VB}=1.5 \mathrm{~V}$. This is again mainly caused by high leakage current which caused temperature effects inside the SiPM reducing the gain. Fig. 4c shows a PDE drop of $25 \%$ from $10 \%$ to $7.5 \%$ PDE. Fig. $4 d$ shows the leakage current increase. We measured a corresponding 60 p.e. RMS noise in a $25 \mathrm{~ns}$ gate at $1.5 \mathrm{~V}$ over voltage at $\mathrm{T}=23^{\circ} \mathrm{C}$. 


\section{$112 \quad 4 . \quad$ Conclusion}

113 Results from a study of the radiation hardness of SiPMs are presented. Recently developed

114 high density ( 10000 cells $\left./ \mathrm{mm}^{2}\right)$ SiPMs from two manufacturers (Hamamatsu and FBK) were 115 exposed to extremely high doses up to $2.2 \times 10^{14} \mathrm{~cm}^{-2} 1 \mathrm{MeV}$ equivalent neutrons $/ \mathrm{cm}^{2}$. The 116 measurements were carried out for the purpose of determining radiation hardness under the 117 extreme conditions expected at the SLHC. We found that the samples are still operational at this 118 dose especially if cooled to around $\mathrm{T}=5^{\circ} \mathrm{C}$. The main problems we found for operation at room 119 temperature are very high noise, breakdown voltage shift, and gain drift due to self heating of 120 the devices. To reach higher radiation tolerance we propose to reduce the SiPM gain and work 121 on electric field shaping. We are now developing even smaller cell devices (7.5 and 5 micron) in collaboration with Irst-FBK to even further reduce the gain.

\section{Acknowledgments}

The authors would like to express their gratitude to the LIF irradiation facility personel (especially to E. Cortina from AIDA project) and the CERN PH/DT IRRAD Facility team (M. Glaser and F. Ravotti) for their help in irradiations and hospitality. The research leading to these results has received funding from the European Commission under the FP7 Research Infrastructures project AIDA, grant agreement no. 262025. This work was supported by the

130 U.S. National Science Foundation and by the Russian Ministry of Education (Russian state grant RFMEFI61014X0004).

\section{References}

134 2. Y. Musienko, D. Renker, S. Reucroft et al., Nucl. Instr. and Meth. A 581 (2007) 433.

135 3. A. Bohn et al., Nucl. Instr. and Meth. A 598 (2009) 722.

136 4. S. Sánchez Majos et al., Nucl. Instr. and Meth. A 602 (2009) 506.

7. Y. Musienko et al., Nucl. Instr. and Meth. A 610 (2009) 87.

140 8. A. Heering et al.,Radiation Damage studies on SiPMs for calorimertry at the super LHC, 2007 IEEE Nuclear Science Symposium Conference Record, Vol. 2, 1523-1526 (2008).

142 9. Y. Musienko, A. Heering, R. Ruchti et al., Nucl. Instr. and Meth. A 787 (2015) 319.

143 10. M. Moll, Radiation Damage in Silicon Particle Detectors, Ph.D. Thesis, Hamburg University, 1999, DESYTHESIS-1999-040, ISSN-1435-8085, (http://mmoll.web.cern.ch/mmoll/thesis/)

145 11. G. Vincent, A. Chantre and D. Bois, J. Appl. Phys. 50 (1979) 5484.

146 12. P.A. Martin, B.G. Streetman and K. Hess, J. Appl. Phys. 52 (1982) 7409. 
Figure captions:

156 Fig. 1. Projected noise for an "ideal" SiPM with thickness of $2 \mu \mathrm{m}$ and GF of $30 \%$ vs. actual data of HPK 15 micron SiPM.

Fig. 2.10 micron cell pitch HPK SiPM with $\emptyset 2.8 \mathrm{~mm}$ after a total dose of $1.210^{13} \mathrm{n} / \mathrm{cm}^{2}$. a) Dark current vs. bias voltage. b) Dark current vs. over-voltage.

Fig. 3. 10 micron cell HPK 8 channel array with $\emptyset 2.8 \mathrm{~mm}$ SiPMs with a maximum dose of $2.210^{14} \mathrm{n} / \mathrm{cm}^{2}$ in channel 2. a) Dark current vs. bias voltage. b) Bias voltage shift vs. dark current at gain 1 .

Fig. 4. 12 micron cell $1 \mathrm{~mm}^{2}$ FBK diode after a dose of $2 * 10^{11}$ and $2.2 * 10^{14} \mathrm{n} / \mathrm{cm}^{2}$. a)

164 Response vs. Bias voltage. b) Response vs. over-voltage. c) PDE (proportional to number of p.e.) vs. over-voltage. d) Leakage current vs. over-voltage. 


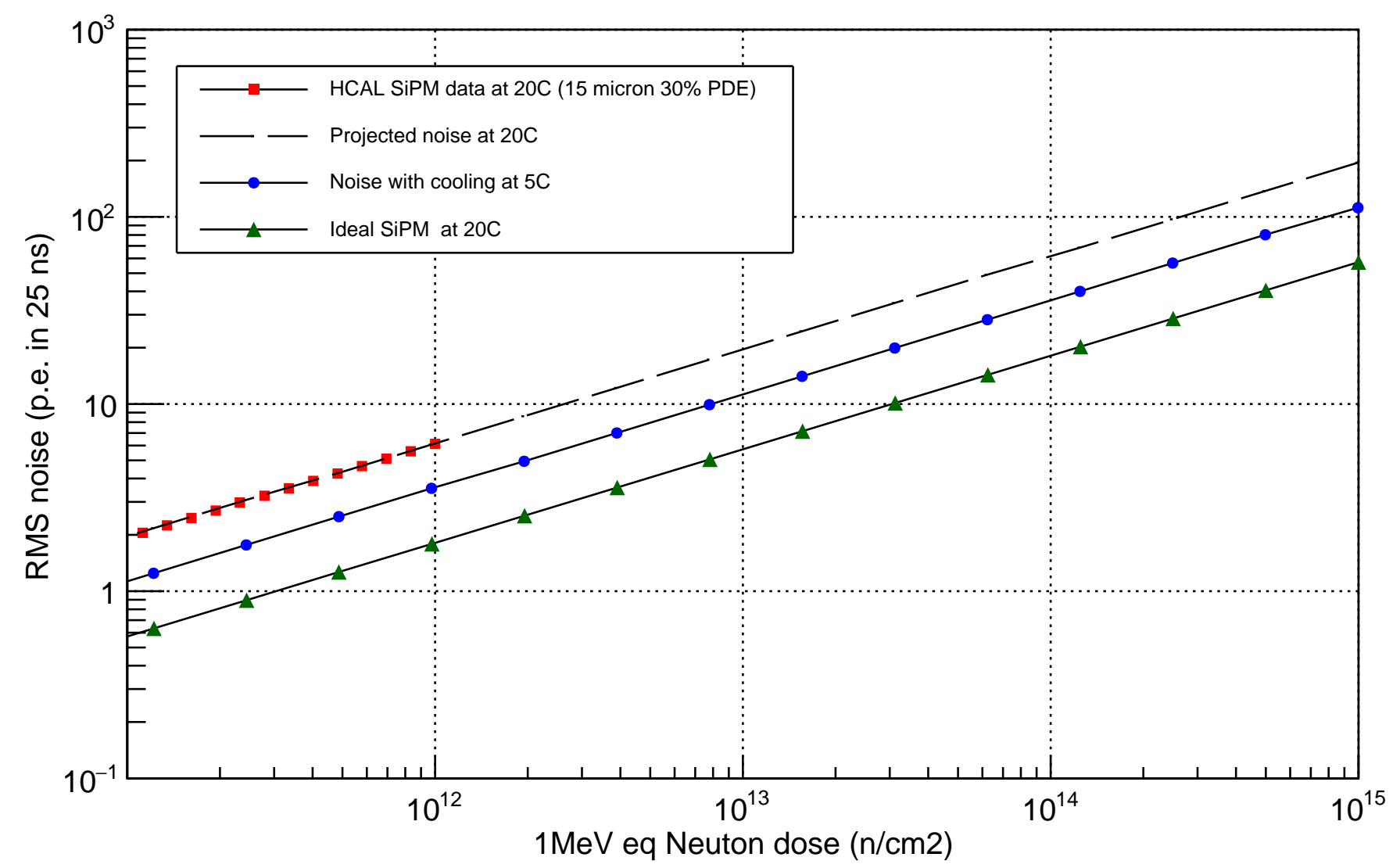




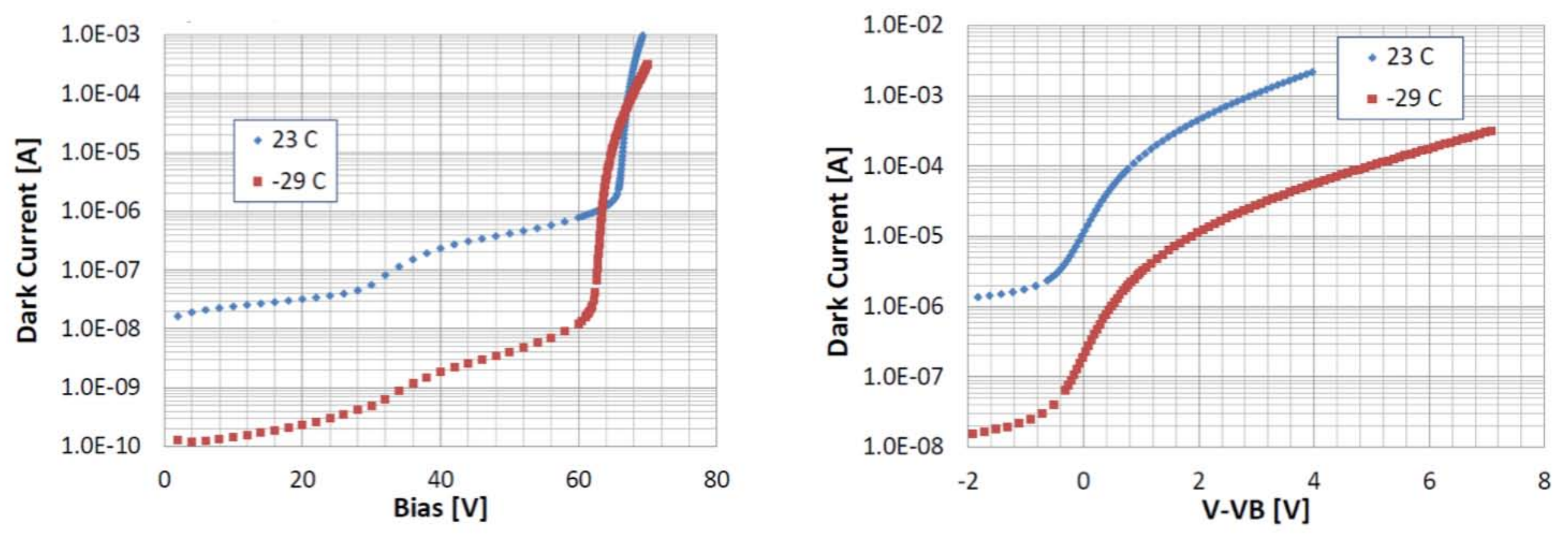



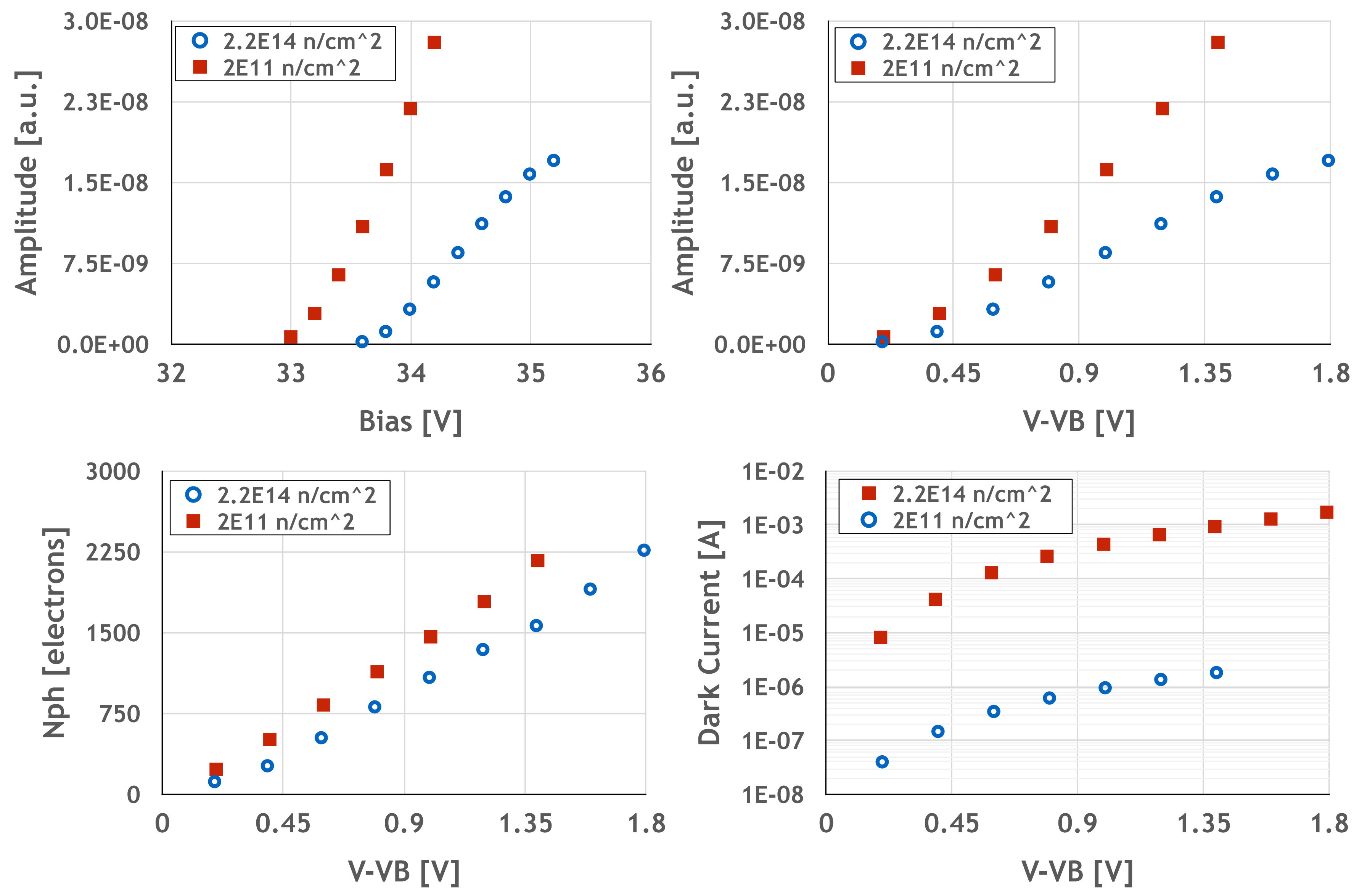